\title{
Clinical efficacy of regional citrate anticoagulation in continuous renal replacement therapy: systematic review and meta-analysis
}

\author{
Hong Chang, Yueying Gong, Caixia Li, Zengwen Ma \\ EICU, Qinghai Provincial People's Hospital, Xining, China \\ Contributions: (I) Conception and design: H Chang, Z Ma; (II) Administrative support: Y Gong; (III) Provision of study materials or patients: H \\ Chang, Y Gong, C Li; (IV) Collection and assembly of data: All authors; (V) Data analysis and interpretation: H Chang, C Li, Z Ma; (VI) Manuscript \\ writing: All authors; (VII) Final approval of manuscript: All authors. \\ Correspondence to: Zengwen Ma. EICU, Qinghai Provincial People's Hospital, No. 2, Gonghe Road, Chengdong District, Xining, China. \\ Email: Yingying20080216@163.com.
}

\begin{abstract}
Background A systematic review and meta-analysis were conducted to explore the clinical efficacy and coagulation function of regional citrate anticoagulation (RCA) in continuous renal replacement therapy (CRRT) in critically ill patients, to provide an effective treatment options for CRRT in severe patients.

Methods: The English databases Embase, Medline, PubMed, Ovid, Springer, and Web of Science were searched to screen for randomized controlled trials (RCTs) on RCA in the CRRT treatment of critically ill patients published before June 1, 2020. Meta analysis using the RevMan5.3 provided by the Cochrane collaboration network. The search terms included "citrate anticoagulation", "patient in severe condition", "CRRT", "clinical effect", and "coagulation function".

Results: ten articles meeting requirements were included, comprising 1,411 subjects. Meta-analysis results showed that after treatment, total calcium/ionized calcium (totCa/ionCa) [mean difference $(\mathrm{MD})=0.05 ; 95 \%$ confidence interval (CI): $(-0.02$ to 0.12$) ; Z=1.31 ; P=0.19]$, prothrombin time $[M D=4.51 ; 95 \% \mathrm{CI}$ : $(2.77$, 6.24); $\mathrm{Z}=5.10 ; \mathrm{P}<0.00001]$, activated partial thromboplastin time [MD =2.56; $95 \% \mathrm{CI}$ : $(1.17,3.95) ; \mathrm{Z}=3.61$; $\mathrm{P}=0.0003]$, and thrombin time $[\mathrm{MD}=4.22 ; 95 \% \mathrm{CI}:(2.07,6.36) ; \mathrm{Z}=3.85 ; \mathrm{P}=0.0001]$ all increased. However, platelet count $[\mathrm{MD}=-5.75 ; 95 \% \mathrm{CI}:(-8.85,-2.64) ; \mathrm{Z}=3.63 ; \mathrm{P}=0.0003]$, cystatin $[\mathrm{MD}=-0.39 ; 95 \% \mathrm{CI}:(-0.63$, $-0.15) ; Z=3.22 ; P=0.001]$, alanine aminotransferase $[M D=-17.63 ; 95 \% \mathrm{CI}:(-20.09,-15.16) ; Z=14.02$; $\mathrm{P}<0.00001]$, aspartate aminotransferase $[\mathrm{MD}=-6.49 ; 95 \% \mathrm{CI}:(-11.94,-1.04) ; \mathrm{Z}=2.33 ; \mathrm{P}=0.02]$, creatinine $[\mathrm{MD}=-3.70 ; 95 \% \mathrm{CI}:(-5.08,-2.32) ; \mathrm{Z}=5.24 ; \mathrm{P}<0.00001]$, and total bilirubin $[\mathrm{MD}=-3.65 ; 95 \% \mathrm{CI}$ : $(-5.91$, -1.40); $Z=3.18 ; \mathrm{P}=0.001$ ] all decreased. Except for totCa/ionCa, the differences in other indicators were not statistically significant compared with the control group.
\end{abstract}

Discussion: RCA can significantly improve the clinical efficacy and blood coagulation indicators of CRRT for severely ill patients.

Keywords: Citrate; critically ill patients; continuous renal replacement therapy (CRRT); clinical efficacy; coagulation function

Submitted Jun 09, 2021. Accepted for publication Jul 23, 2021.

doi: 10.21037/apm-21-1693

View this article at: https://dx.doi.org/10.21037/apm-21-1693

\section{Introduction}

Continuous renal replacement therapy (CRRT) refers to blood purification treatment that continuously and slowly removes water and solutes through extracorporeal blood purification to replace kidney function (1). CRRT prolongs the blood purification treatment time and reduces the treatment efficiency per unit time compared with ordinary hemodialysis (2). It can also minimize the impact of solute concentration and volume changes in the blood on the body and uses filters with high permeability and 
good biocompatibility $(3,4)$, which provides an extremely important homeostatic balance for the treatment of critically ill patients. Moreover, CRRT strives to utilize the best treatment window for the primary diseases of critically ill patients (5). Since CRRT is a continuous and long-term course of treatment, anticoagulation of blood in extracorporeal circulation is a very tricky problem. Effective anticoagulation to ensure the circulation of blood is an important measure for the continuous implementation of CRRT. Anticoagulation technology is the basic condition for CRRT to extend the filter duration and maintain the unobstructed cardiopulmonary bypass, which is closely related to the treatment time and clinical efficacy (6). Reasonable anticoagulation should follow the principle of individualization, the correct selection of anticoagulants and close clinical monitoring are very important to prevent complications. Therefore, which anticoagulation method the patient chooses during treatment is particularly important.

Heparin is the most common anticoagulant in clinical practice, which is a mucopolysaccharide containing sulfuric acid group. As an anticoagulant, it is a polymer formed by alternately connecting two polysaccharides. It is a natural anticoagulant substance in animals. Heparin has anticoagulant effects both in vivo and in vitro. Low molecular weight heparin has strong anticoagulant effect and is not easy to cause bleeding, which can avoid bleeding when applied in high-risk bleeding patients. Clinically, it is adopted for thromboembolic diseases, myocardial infarction, cardiovascular surgery, etc. (7). After clinical use, it has been found that its anticoagulant effect is good, but it can easily cause spontaneous bleeding, manifesting as mucosal bleeding, hemorrhage in the joint cavity, and bleeding from wounds. Regional citrate anticoagulation (RCA) refers to the application of citric acid for external anticoagulation during hemodialysis. This kind of anticoagulation method involves in vitro anticoagulation, and the anticoagulant does not enter the body, which greatly reduces the influence of the anticoagulant on the risk of bleeding in the body. However, the operation of this method is complicated, and the clinical application is relatively small, unless certain patients with a bleeding tendency are encountered (8). It is urgent to find effective anticoagulant treatment methods that can be applied to the CRRT process to ensure the treatment effect of patients, and correct selection of anticoagulants and close clinical monitoring are very important to prevent complications. To further study the clinical efficacy and coagulation function of RCA in the CRRT treatment of critically ill patients, the clinical randomized controlled trials (RCTs) on RCA for CRRT in critically ill patients were screened from the literature. In this study, ten randomized controlled clinical trials of CRRT critically ill patients with RCA were screened for meta-analysis. The clinical efficacy of anticoagulant citrate in CRRT in critically ill patients was quantitatively and systematically analyzed. This research aimed to provide a theoretical basis for evaluating the clinical efficacy and coagulation function of RCA in the CRRT treatment of critically ill patients.

We present the following article in accordance with the PRISMA reporting checklist (available at https://dx.doi. org/10.21037/apm-21-1693).

\section{Methods}

\section{Screening criteria}

Inclusion criteria: (I) research subjects were patients with severe CRRT; (II) the research type was RCTs published in English databases, and the language was English; (III) the treatment for experimental group was RCA that for control was heparin, the basic inclusion data of two groups are similar and contain relevant indicators; (IV) the evaluation indicator of the study outcome was the patient's blood coagulation function [total calcium/ ionized calcium (totCa/ionCa) ratio, prothrombin time, activated partial thromboplastin time, thrombin time, platelet count, cystatin, alanine aminotransferase, aspartate aminotransferase, creatinine, and total bilirubin].

Exclusion criteria: (I) research types that were non-RCTs; (II) research objects were animals or cells; (III) unpublished literature; (IV) the operation of the experimental group was non-glucocorticoid; (V) research subjects were noncritically ill patients; (VI) the included data is not compliant and incomplete to calculate the results based on the data.

\section{Literature search}

Six English databases (PubMed, Embase, Medline, Ovid, Springer, and Web of Science) were searched, and the search deadline was December 31, 2020. Publicly published clinical RCTs of RCA for CRRT in critically ill patients were screened. Subject terms and keywords were searched, including: "citrate anticoagulation", "patient in severe condition", "CRRT", "clinical effect", and "coagulation function". The terms "and" or "or" were used for joint 
searches among terms. Two researchers independently implemented the searching works.

\section{Screening}

Two researchers do screen independently. NoteExpress 3.2 was employed to establish a database, then duplicates of the retrieved literature were eliminated. After duplicates were removed, searching for the required information based on the article summary and keywords, the two researchers screened the documents that met the inclusion criteria and excluded the article according to the exclusion criteria. After the manual literature screening, inclusion of the literature was determined according to the criteria mentioned above. If the two researchers disagreed, they could discuss and resolve the different opinions. If there is any objection to the results of the article, please ask a third researcher to screen and exclude the article.

\section{Data extraction}

The two researchers conducted bias analysis, screened the article data, and included the basic data that contained the characteristics of the basic research objects, including: The first author, published year, group, sample size, counter measure, records into a form. Independently, two researchers finished the extraction and cross-examined after that. If there was a disagreement, it would be discussed and resolved. If the opinions were different, it would be decided by a third party after arbitration. The data extracted from the documents that met the inclusion criteria mainly included: (I) the title of the document, the first author (one name), the publication time, and the research region; (II) research subjects' ages, sample size, and baseline data comparability; (III) research plan design, implementation methods, intervention measures and control measures, and anti-bias measures; (IV) research outcome indicators and research data.

\section{Quality assessment}

The risk of bias of the original included articles was assessed via Cochrane Handbook for systematic reviews of intervention 5.0.2 regarding the generation of random sequence, the blinding of patients and experimenters, the blinding for outcome assessors, complete data or not, results were selective reporting or not, and other sources of bias were found or not. If the two researchers had inconsistent results of the literature evaluation, the results would be discussed, or dealt by a third party.

\section{Statistical methods}

The risk of literature bias was assessed using Cochrane intervention system review manual 5.0.2. Stata 11.0 was used to collect the included literatures. RevMan5.3 was used for meta-analysis of the combined statistical data, and the forest map and funnel map were drawn. The relative risk (RR) was used as effect quantity of binary variables in the postoperative adverse reaction count data. Then, $95 \%$ confidence interval (CI) and continuous variables such as heart rate (HR), mean arterial pressure (MAP) and visual analogue scale (VAS) were calculated. Mean difference (MD) is set as effect quantity if the unit of detection index is the same. When we can combine the research results of various literatures, a meta-analysis is performed. The heterogeneity of the included articles was evaluated by $\mathrm{I}^{2}$ test. The greater the $\mathrm{I}^{2}$, the greater the heterogeneity. If $\mathrm{I}^{2}>50 \%$ and the source of heterogeneity could not be explained, the random effects model (REM) was used for meta-analysis. If $\mathrm{I}^{2}<50 \%$, that is to say, the heterogeneity of literature is good, the fixed effects model (FEM) combined with effect quantity is utilized. If there are less than two items in the study, descriptive analysis is used. The combined effects were measured by $\mathrm{U}$ test and $95 \% \mathrm{CI}$ test. $\mathrm{U}$ test results were expressed by $\mathrm{P}$, and $\mathrm{P}<0.05$ was statistically considerable. Binary variables were tested with $95 \%$ CI. When $95 \%$ CI $>1$ or $<1$, the difference was substantial. When $95 \%$ CI was 1 , the difference was not considerable. Ninety-five percent CI was used to test continuous variables. When $95 \% \mathrm{CI}>0$ or $<0$, the difference was considerable.

\section{Results}

\section{Screening results}

After a preliminary search, a total of 1,226 relevant documents were screened in the preliminary review. Among them, 431 related documents were retrieved from PubMed, 251 related documents were retrieved from Embase, 113 related documents were retrieved from Medline, 156 related documents were retrieved from Springer, 145 related documents were retrieved from Ovid, and 130 related documents were retrieved from Web of Science. All 1,226 article titles were imported into NoteExpress 3.2 after the preliminary search was completed, and 185 documents 


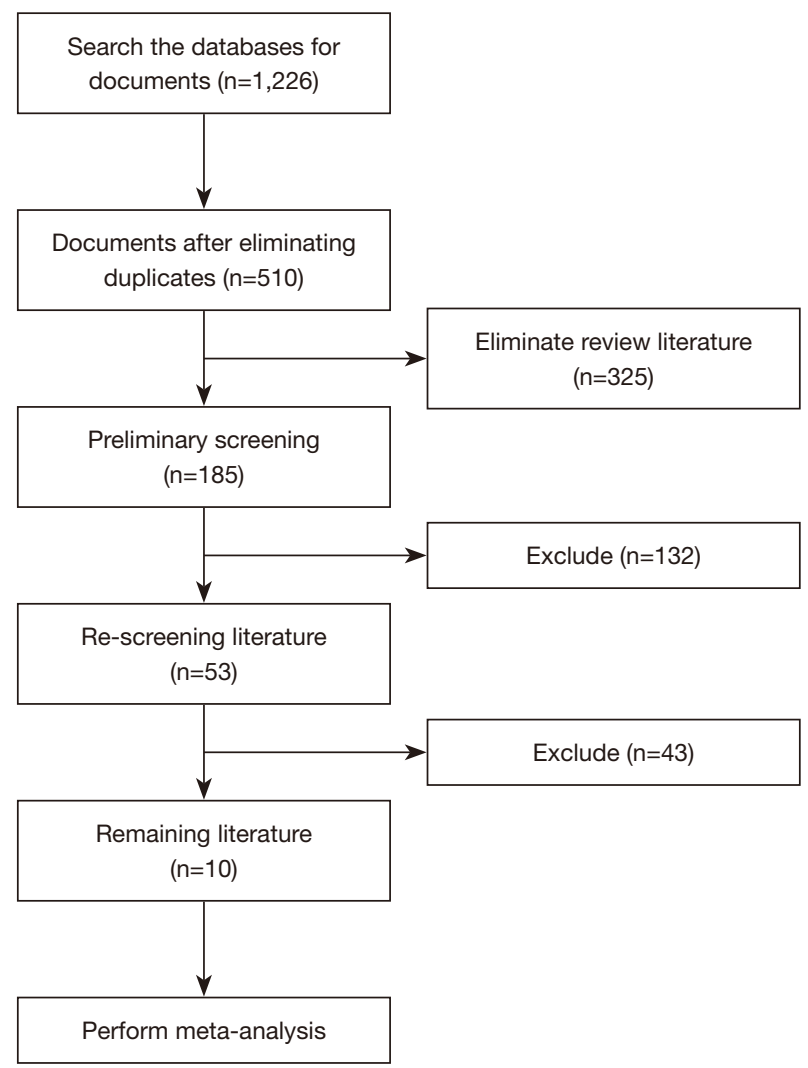

Figure 1 Literature retrieval process.

remained after the duplicates were eliminated. Two researchers then read the titles and abstracts and screened the literature. After screening, there were 53 remaining documents. Then, two researchers cross-examined the texts, and then screened and excluded the literature. Ten articles were included finally (Figure 1 and Table 1). They were all publicly published RCTs, and the publication times were before 2020. The 10 articles included a total of 1,298 research subjects, and information such as the age of patients in two groups were comparable.

\section{Risk of bias assessment results}

Bias risk map was plotted by RevMan5.3. The risk bias assessment items of this study include: (I) random sequence generation. The 10 articles (9-18) described the specific grouping as "according to the operation method", indicating low risk. (II) Assign hidden. None of the 10 documents mentioned presence of "funding concealment", indicating that the risk was not clear. (III) Blinding of subjects: 6 of 10 documents $(9-11,13-15)$ mentioned that "the patient knew and signed the informed consent", but didn't clarify blinding of experimenter (unclear risk). (IV) Blinding of outcome assessors: 10 articles did not mention it, suggesting unclear risk. (V) Results data integrity: the results of 10 articles were complete, indicating low risk. (VI) Selective report: 10 articles without selective report, low risk. (VII) Other risk of bias: in 10 articles, the number of subjects in the two groups was not consistent, indicating high risk. The results of deviation risk assessment are shown in Figures 2,3.

\section{Ratio of totCa/ionCa}

Five works $(9,10,13,14,16)$ analyzed the totCa/ionCa of patients after treatment. A total of 907 severe CRRT patients were enrolled, there were 357 experimental cases and 550 controls. Heterogeneity test were $\mathrm{I}^{2}=62 \%$ and $\mathrm{P}=0.03$, indicating certain heterogeneity in these studies. Therefore, REM was adopted, and the results were illustrated in Figure 4. Combined effect was MD $=0.05 ; 95 \%$ CI: $(-0.02,0.12) ; \mathrm{Z}=1.31 ; \mathrm{P}=0.19$. The diamonds are to the right of vertical line (VL) in the forest plot, suggesting that total ion ratio is RCA in CRRT of critically ill patients was superior to controls.

\section{Protbrombin time}

Five works $(9,10,15-17)$ analyzed prothrombin time. Eight hundred and eighty-six patients with severe CRRT were enrolled, with 343 as experimental subjects and 543 as controls. The heterogeneity test result suggested that $\mathrm{I}^{2}=98 \%, \mathrm{P}<0.00001$, and there was heterogeneity. Therefore, we use REM for analysis (Figure 5). The combined effect was $\mathrm{MD}=4.51 ; 95 \% \mathrm{CI}:(2.77,6.24)$; $\mathrm{Z}=5.10 ; \mathrm{P}<0.00001$. The diamond on the right side of $\mathrm{VL}$ shows that the prothrombin time of critically ill patients receiving RCA treatment in CRRT is longer than that of controls.

\section{Activated partial thromboplastin time}

Four works $(9-11,18)$ analyzed the partial thrombin activation time of patients. There were 330 patients with severe CRRT, including 142 experimental cases and 188 controls. The heterogeneity test result was $\mathrm{I}^{2}=92 \%$, $\mathrm{P}<0.00001$, and heterogeneity was indicated. Therefore, we use REM for analysis (Figure 6). The combined effect was $M D=2.56 ; 95 \%$ CI: $(1.17,3.95) ; Z=3.61 ; P=0.0003$. The diamond is on right side of VL, which means that the 
Table 1 Basic characteristics of the included studies

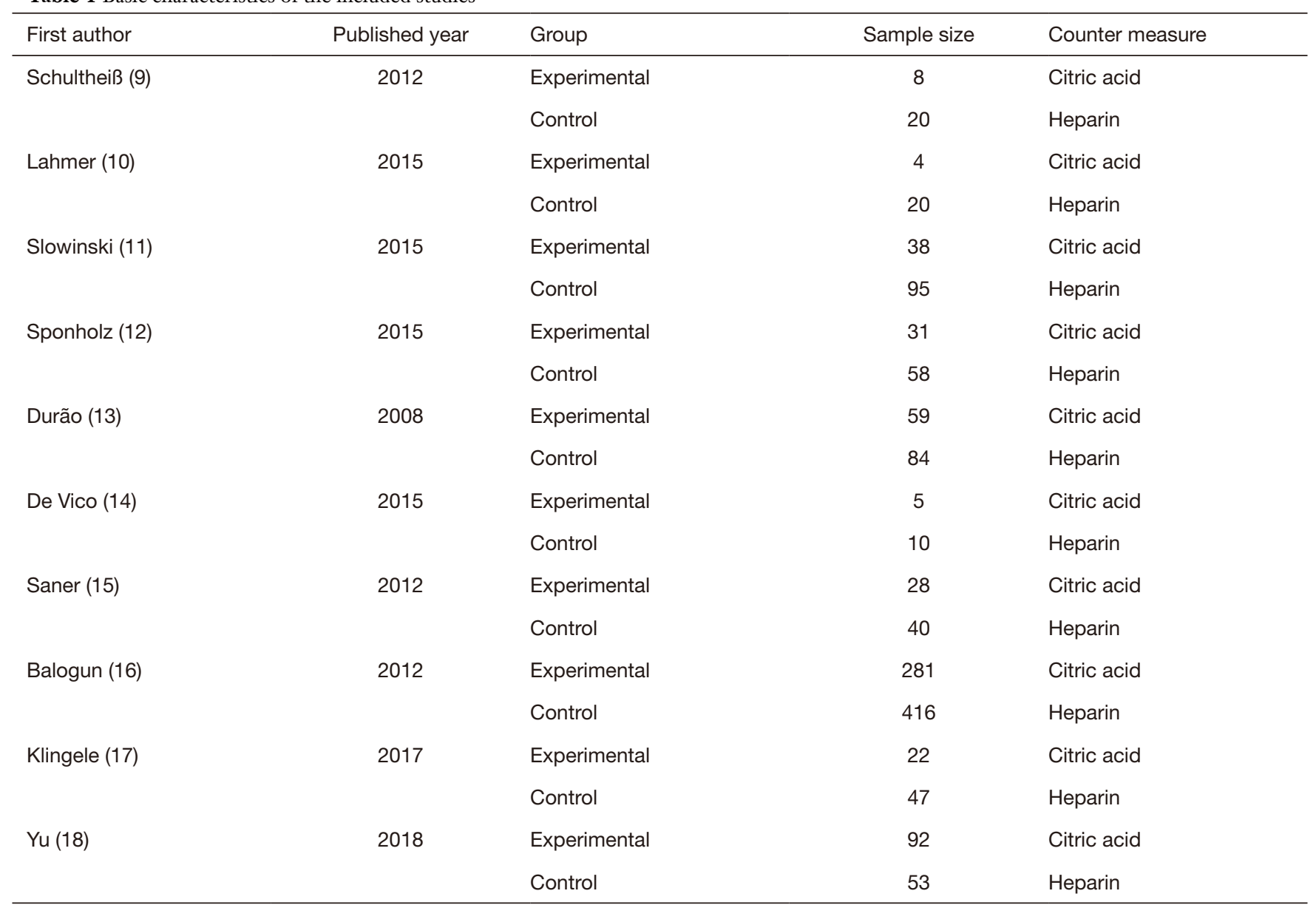

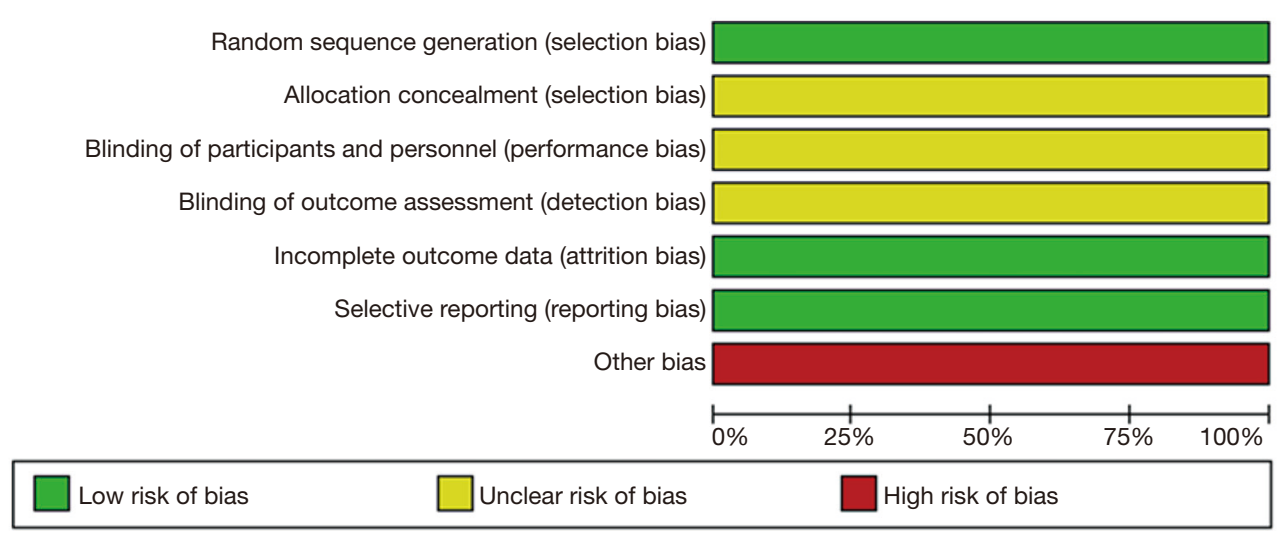

Figure 2 Bar graph of bias assessment of the included literature. 


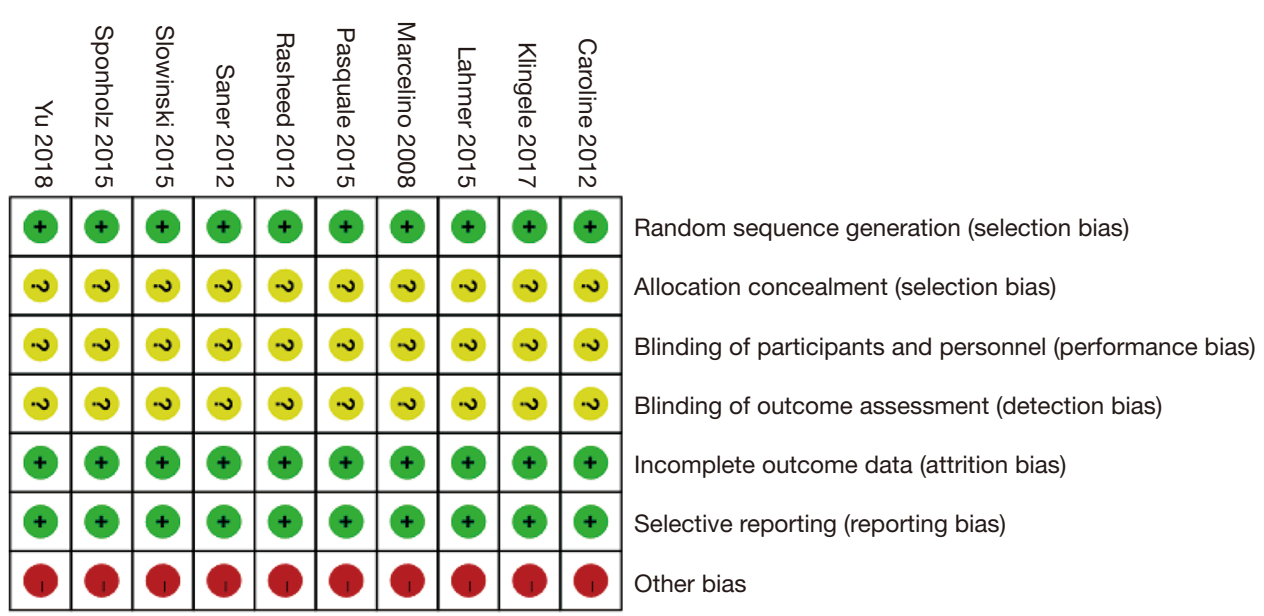

Figure 3 Assessment results of the risk of bias in the included literature.

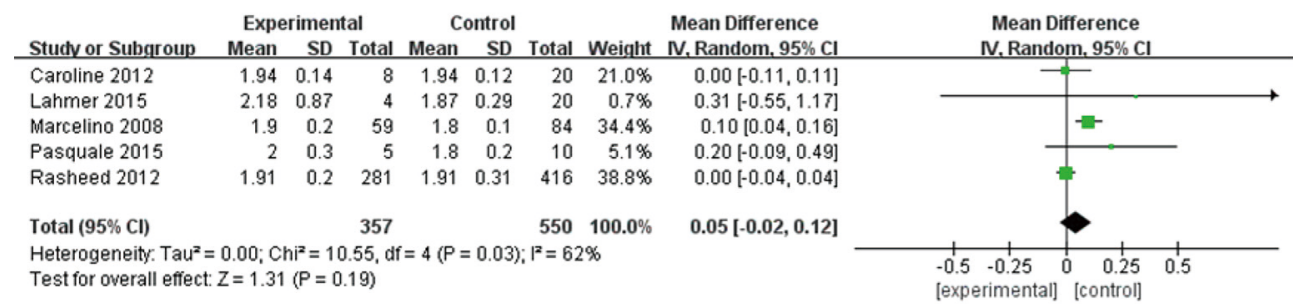

Figure 4 Forest plot of the totCa/ionCa ratio of critically ill patients treated by RCA in CRRT. totCa/ionCa, total calcium/ionized calcium; RCA, regional citrate anticoagulation; CRRT, continuous renal replacement therapy; SD, standard deviation; CI, confidence interval.

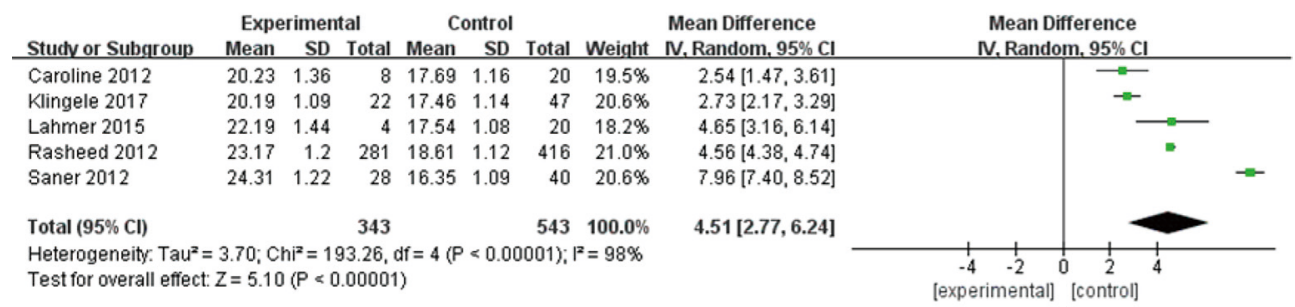

Figure 5 Forest plot of the prothrombin time of critically ill patients treated by RCA in CRRT. RCA, regional citrate anticoagulation; CRRT, continuous renal replacement therapy; SD, standard deviation; CI, confidence interval.

partial thrombin time of critically ill patients treated with RCA activated CRRT is superior to that of controls.

\section{Thrombin time}

Six works $(9-11,15,17,18)$ analyzed the thrombin time of 467 patients with severe CRRT, with 192 experimental cases and 275 controls. The heterogeneity test result shows that $\mathrm{I}^{2}=95 \%$ and $\mathrm{P}<0.00001$, and there was heterogeneity.
Therefore, we use REM for analysis, and the results are plotted in Figure 7. Combined effect revealed that $\mathrm{MD}=4.22 ; 95 \% \mathrm{CI}$ : (2.07, 6.36); $\mathrm{Z}=3.85 ; \mathrm{P}=0.0001$. The diamond is on the right side of VL, and RCA in CRRT leads to the prolongation of thrombin time in critically ill patients relative to controls.

\section{Platelet count}

Four works $(9,10,13,18)$ analyzed the platelet count of 


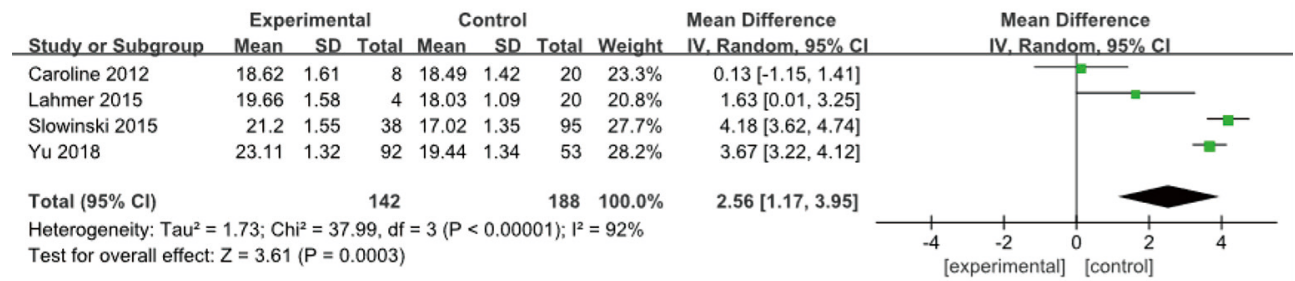

Figure 6 Forest plot of the activated partial thromboplastin time of critically ill patients treated by RCA in CRRT. RCA, regional citrate anticoagulation; CRRT, continuous renal replacement therapy; SD, standard deviation; CI, confidence interval.

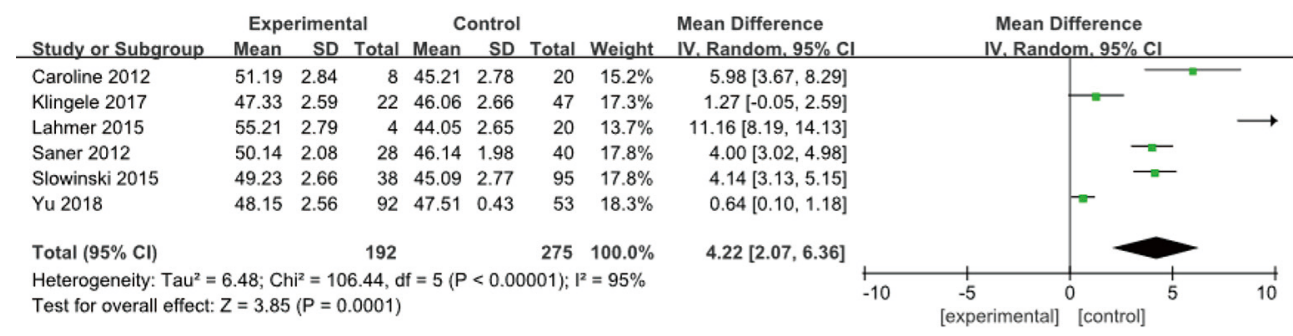

Figure 7 Forest plot of the thrombin time of critically ill patients treated by RCA in CRRT. RCA, regional citrate anticoagulation; CRRT, continuous renal replacement therapy; SD, standard deviation; CI, confidence interval.

\begin{tabular}{|c|c|c|c|c|c|c|c|c|c|c|c|}
\hline \multirow[b]{2}{*}{ Study or Subgroup } & \multicolumn{3}{|c|}{ Experimental } & \multicolumn{2}{|c|}{ Control } & \multirow{2}{*}{ Total } & \multirow{2}{*}{\multicolumn{2}{|c|}{$\begin{array}{cc} & \text { Mean Difference } \\
\text { Weight } & \text { N. Random, } 95 \% \mathrm{Cl} \\
\end{array}$}} & \multirow{2}{*}{\multicolumn{2}{|c|}{$\begin{array}{c}\text { Mean Difference } \\
\text { IV. Random, } 95 \% \mathrm{Cl}\end{array}$}} & \\
\hline & Mean & SD & Total & Mean & SD & & & & & & \\
\hline Caroline 2012 & 124.57 & 9.38 & 8 & 125.32 & 9.75 & 20 & $12.3 \%$ & $=0.75[-8.53,7.03]$ & & & \\
\hline Lahmer 2015 & 123.48 & 8.23 & 4 & 126.45 & 8.57 & 20 & $9.9 \%$ & $-2.97[-11.87,5.93]$ & & & \\
\hline Marcelino 2008 & 122.34 & 7.44 & 59 & 127.49 & 7.48 & 84 & $40.3 \%$ & $-5.15[-7.63,-2.67]$ & & & \\
\hline Yu 2018 & 118.67 & 8.4 & 92 & 127.43 & 8.29 & 53 & $37.5 \%$ & $-8.76[-11.58,-5.94]$ & & & \\
\hline Total $(95 \% \mathrm{Cl})$ & & & 163 & & & 177 & $100.0 \%$ & $-5.75[-8.85,-2.64]$ & & & \\
\hline $\begin{array}{l}\text { Heterogeneity. Tau }{ }^{2} \\
\text { Test for overall effec }\end{array}$ & $\begin{array}{l}4.62 ; \mathrm{Ch} \\
\mathrm{Z}=3.63\end{array}$ & $\begin{array}{l}I^{2}=6.2 \\
(P=0 .\end{array}$ & $\begin{array}{l}5, \mathrm{df}= \\
0003)\end{array}$ & $3(\mathrm{~F}$ & & $\%$ & & & $\begin{array}{ll}-10 & -5 \\
\text { [experimental] }\end{array}$ & $\begin{array}{cc}0 & 5 \\
\text { 1] [control] }\end{array}$ & 10 \\
\hline
\end{tabular}

Figure 8 Forest plot of platelet counts of critically ill patients treated by RCA in CRRT. RCA, regional citrate anticoagulation; CRRT, continuous renal replacement therapy; SD, standard deviation; CI, confidence interval.

340 patients with severe CRRT, with 163 experimental cases and 177 controls. The heterogeneity test result was $\mathrm{I}^{2}=52 \%$, $\mathrm{P}=0.10$, and there was heterogeneity. Therefore, we use REM for analysis, as illustrated in Figure 8. Combined effect MD $=-5.75 ; 95 \%$ CI: $(-8.85,-2.64) ; Z=3.63 ; \mathrm{P}=0.0003$. The diamond in the forest diagram is on the left side of $\mathrm{VL}$, and the platelet count of critically ill patients receiving RCA treatment in CRRT is inferior to controls.

\section{Cystatin}

Four articles $(9,11,13,16)$ analyzed the cystatin level of 1,001 patients with severe CRRT, including 386 experimental cases and 615 controls. The heterogeneity test result was $\mathrm{I}^{2}=94 \%$,
$\mathrm{P}<0.00001$, and there was heterogeneity. Therefore, we use REM for analysis, and the outcome is shown in Figure 9. The combined effect was $\mathrm{MD}=-0.39 ; 95 \% \mathrm{CI}:(-0.63,-0.15)$; $Z=3.22 ; P=0.001$. The diamond is on the left side of VL, and the cystatin level of critically ill patients receiving RCA treatment in CRRT is inferior to controls.

\section{Alanine aminotransferase}

Three works $(9,11,15)$ analyzed the alanine aminotransferase level of 229 patients with severe CRRT, including 74 experimental cases and 155 controls. The heterogeneity test result was $\mathrm{I}^{2}=0 \%, \mathrm{P}=0.66$, and there was no heterogeneity. Therefore, the finite element method is used, as plotted 


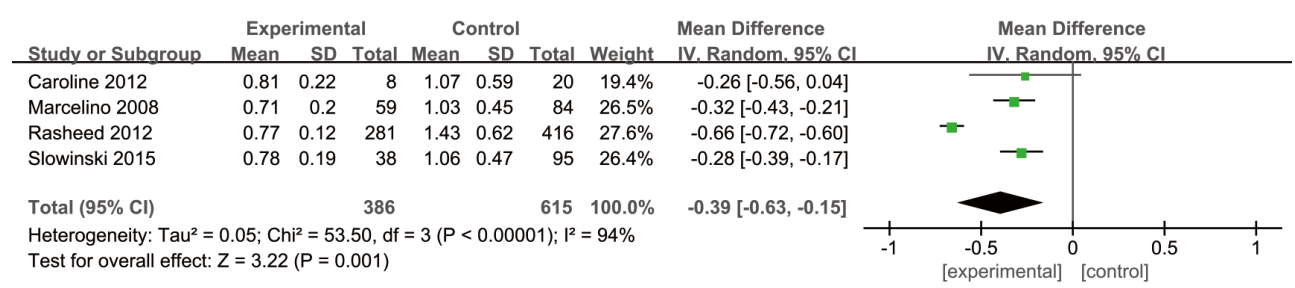

Figure 9 Forest plot of cystatin levels of critically ill patients treated by RCA in CRRT. RCA, regional citrate anticoagulation; CRRT, continuous renal replacement therapy; SD, standard deviation; CI, confidence interval.

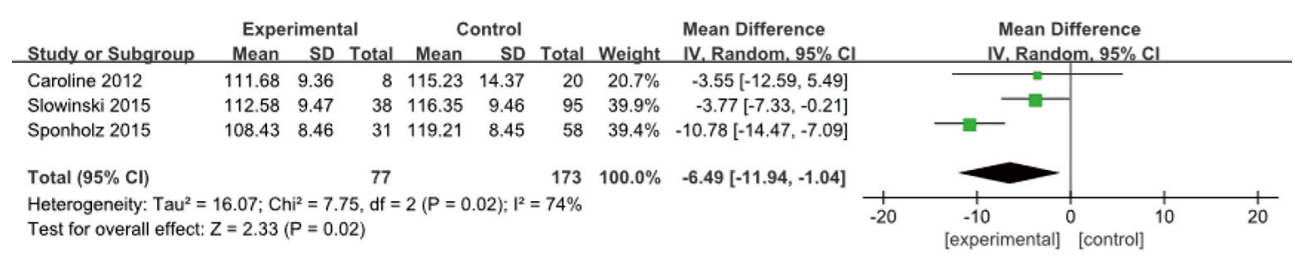

Figure 10 Forest plot of alanine aminotransferase levels of critically ill patients treated by RCA in CRRT. RCA, regional citrate anticoagulation; CRRT, continuous renal replacement therapy; SD, standard deviation; CI, confidence interval.

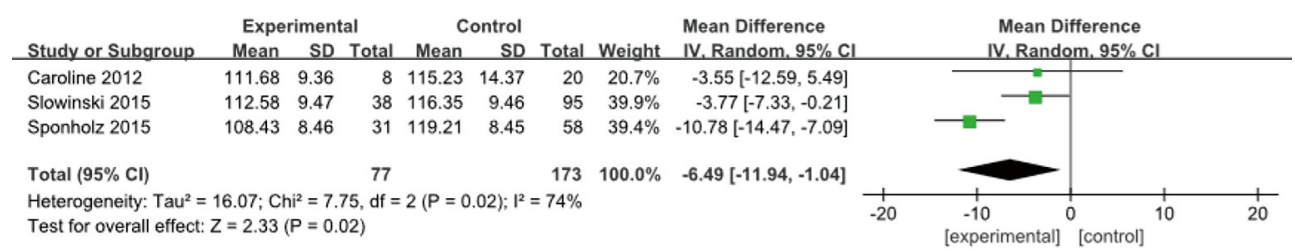

Figure 11 Forest plot of aspartate aminotransferase levels of critically ill patients treated by RCA in CRRT. RCA, regional citrate anticoagulation; CRRT, continuous renal replacement therapy; SD, standard deviation; CI, confidence interval.

in Figure 10. Combined effect shows that $\mathrm{MD}=-17.63$; 95\% CI: $(-20.09,-15.16) ; Z=14.02 ; P<0.00001$. The rhombus of the forest plot is on left side of VL, and alanine aminotransferase level of critically ill patients treated with RCA in CRRT is inferior to controls.

\section{Aspartate aminotransferase}

Three studies $(9,11,12)$ analyzed the aspartate aminotransferase level of 250 patients with severe CRRT, including 77 experimental cases and 173 controls. The heterogeneity test result was $\mathrm{I}^{2}=74 \%, \mathrm{P}=0.02$, and there was heterogeneity. Therefore, we use REM for analysis, and the results are plotted in Figure 11. The combined effect was $M D=-6.49 ; 95 \% \mathrm{CI}:(-11.94,-1.04) ; \mathrm{Z}=2.33 ; \mathrm{P}=0.02$. The diamond is on the left side of $\mathrm{VL}$ in forest map, and the aspartate aminotransferase level of critically ill patients treated with RCA in CRRT is inferior to controls.

\section{Creatinine}

Three research $(9,11,16)$ analyzed the creatinine level of 858 patients with severe CRRT, including 327 experimental cases and 531 controls. The heterogeneity test result was $\mathrm{I}^{2=} 0 \%, \mathrm{P}=0.94$, and there was no heterogeneity. Therefore, the finite element method is used for analysis, as presented in Figure 12. Combined effect MD $=-3.70 ; 95 \%$ CI: $(-5.08$, -2.32); $Z=5.24 ; P<0.00001$. The diamond is on the left side of VL, and the creatinine level of critically ill patients receiving RCA treatment in CRRT is inferior to controls.

\section{Total bilirubin}

Four works $(9-11,17)$ analyzed the total bilirubin of 


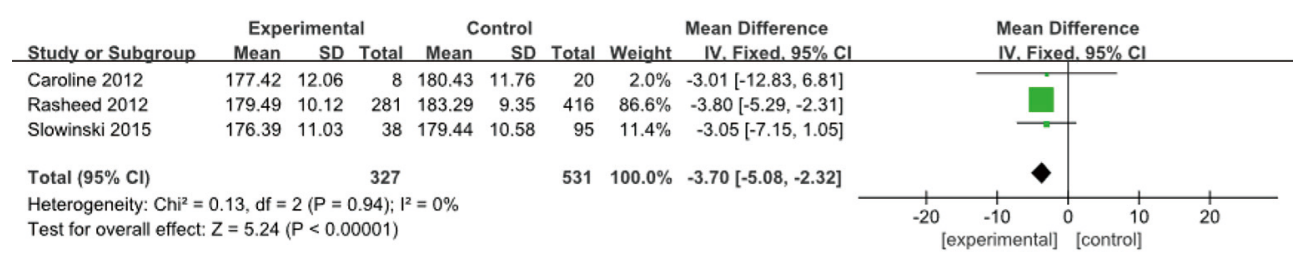

Figure 12 Forest plot of creatinine levels of critically ill patients treated by RCA in CRRT. RCA, regional citrate anticoagulation; CRRT, continuous renal replacement therapy; SD, standard deviation; CI, confidence interval.

\begin{tabular}{|c|c|c|c|c|c|c|c|c|c|c|c|}
\hline \multirow[b]{2}{*}{ Study or Subgroup } & \multicolumn{3}{|c|}{ Experimental } & \multicolumn{2}{|c|}{ Control } & \multirow[b]{2}{*}{ Total } & \multirow[b]{2}{*}{ Weight } & \multirow{2}{*}{$\begin{array}{l}\text { Mean Difference } \\
\text { IV. Random. } 95 \% \mathrm{Cl}\end{array}$} & \multirow{2}{*}{\multicolumn{3}{|c|}{$\begin{array}{c}\text { Mean Difference } \\
\text { IV. Random. } 95 \% \mathrm{cl}\end{array}$}} \\
\hline & Mean & SD & Total & Mean & SD & & & & & & \\
\hline Caroline 2012 & 23.76 & 3.98 & 8 & 24.13 & 4.37 & 20 & $20.7 \%$ & $-0.37[-3.73,2.99]$ & & & \\
\hline Klingele 2017 & 21.33 & 3.77 & 22 & 27.39 & 3.49 & 47 & $30.3 \%$ & $-6.06[-7.92,-4.20]$ & $-\mathrm{m}$ & & \\
\hline Lahmer 2015 & 22.74 & 3.76 & 4 & 24.22 & 5.2 & 20 & $15.9 \%$ & $-1.48[-5.81,2.85]$ & & & \\
\hline Slowinski 2015 & 20.7 & 3.59 & 38 & 25.24 & 4.38 & 95 & $33.1 \%$ & $-4.54[-5.98,-3.10]$ & & & \\
\hline Total $(95 \% \mathrm{Cl})$ & & & 72 & & & 182 & $100.0 \%$ & $-3.65[-5.91,-1.40]$ & & & \\
\hline $\begin{array}{l}\text { Heterogeneity: } \mathrm{Tau}^{2}= \\
\text { Test for overall effect }\end{array}$ & $\begin{array}{l}3.46 ; \mathrm{Cl} \\
\mathrm{Z}=3.18\end{array}$ & $\begin{array}{l}i^{2}=10 \\
(P=0\end{array}$ & $\begin{array}{l}.35, \mathrm{df} \\
.001)\end{array}$ & $=3(\mathrm{P}$ & $=0.02$ & $1^{2}=7$ & & & $\begin{array}{ll}-10 & -5 \\
\text { [experimental] }\end{array}$ & $\begin{array}{cc}0 & 5 \\
& \text { [control] }\end{array}$ & 10 \\
\hline
\end{tabular}

Figure 13 Forest plot of total bilirubin of critically ill patients treated by RCA in CRRT. RCA, regional citrate anticoagulation; CRRT, continuous renal replacement therapy; SD, standard deviation; CI, confidence interval.

254 patients with severe CRRT, including 72 experimental cases and 182 controls. The heterogeneity test result was $\mathrm{I}^{2}=71 \%, \mathrm{P}=0.02$, and there was heterogeneity. Therefore, we use REM for analysis, and the outcome is illustrated in Figure 13. Combined effect $\mathrm{MD}=-3.65 ; 95 \%$ CI: $(-5.91$, $-1.40) ; Z=3.18 ; P=0.001$. The diamond is on the left side of $\mathrm{VL}$, and total bilirubin of critically ill patients treated with RCA in CRRT is inferior to controls.

\section{Publication bias analysis}

The clinical efficacy and coagulation function of RCA in the CRRT treatment of critically ill patients and postoperative adverse reaction indicators were analyzed for publication bias via RevMan5.3, as illustrated in Figure 14. The combined effect of tot $\mathrm{Ca} / \mathrm{ionCa}$, platelet count, alanine aminotransferase, aspartate aminotransferase, creatinine, and total bilirubin basically within credible interval indicate low bias. In the funnel plots of the patients' prothrombin time, activated partial thromboplastin time, thrombin time, and cystatin levels, some points were outside the CI. A certain publication bias was suggested.

\section{Discussion}

Dialysis is often used in clinically severe patients, such as patients with renal failure. However, patients often suffer from malnutrition during treatment, which seriously affects their treatment (19-21). CRRT is a newly developed blood purification technology, which has a powerful function of stabilizing the internal environment and removing inflammatory mediators. In the treatment of severe acute renal failure, multiple organ dysfunction syndrome, severe acute pancreatitis, acute respiratory distress syndrome, and other clinical critical diseases, it has the advantages of high stability, a strong ability to improve blood quality, and a good removal effect of toxic substances (22). Clinically, this method is often used to treat patients with renal failure, relieve renal pressure, correct electrolyte disorders, and remove inflammatory mediators, thereby reducing the impact of the disease (23). CRRT is a continuous and long-term course of treatment, anticoagulation of blood in extracorporeal circulation is a very tricky problem. Effective anticoagulation to ensure the circulation of blood is an important measure for the continuous implementation of CRRT. Anticoagulation technology is the basic condition for CRRT to extend the filter duration and maintain the unobstructed cardiopulmonary bypass. However, if anticoagulation is not performed in the course of treatment, thrombosis can easily develop, toxins cannot be fully removed, and the patient's coagulation function indicators may even change, which ultimately affects the clinical efficacy (24-26). Although frequent filter replacement can relieve blood clotting, it increases the cost of treatment for patients and also affects 

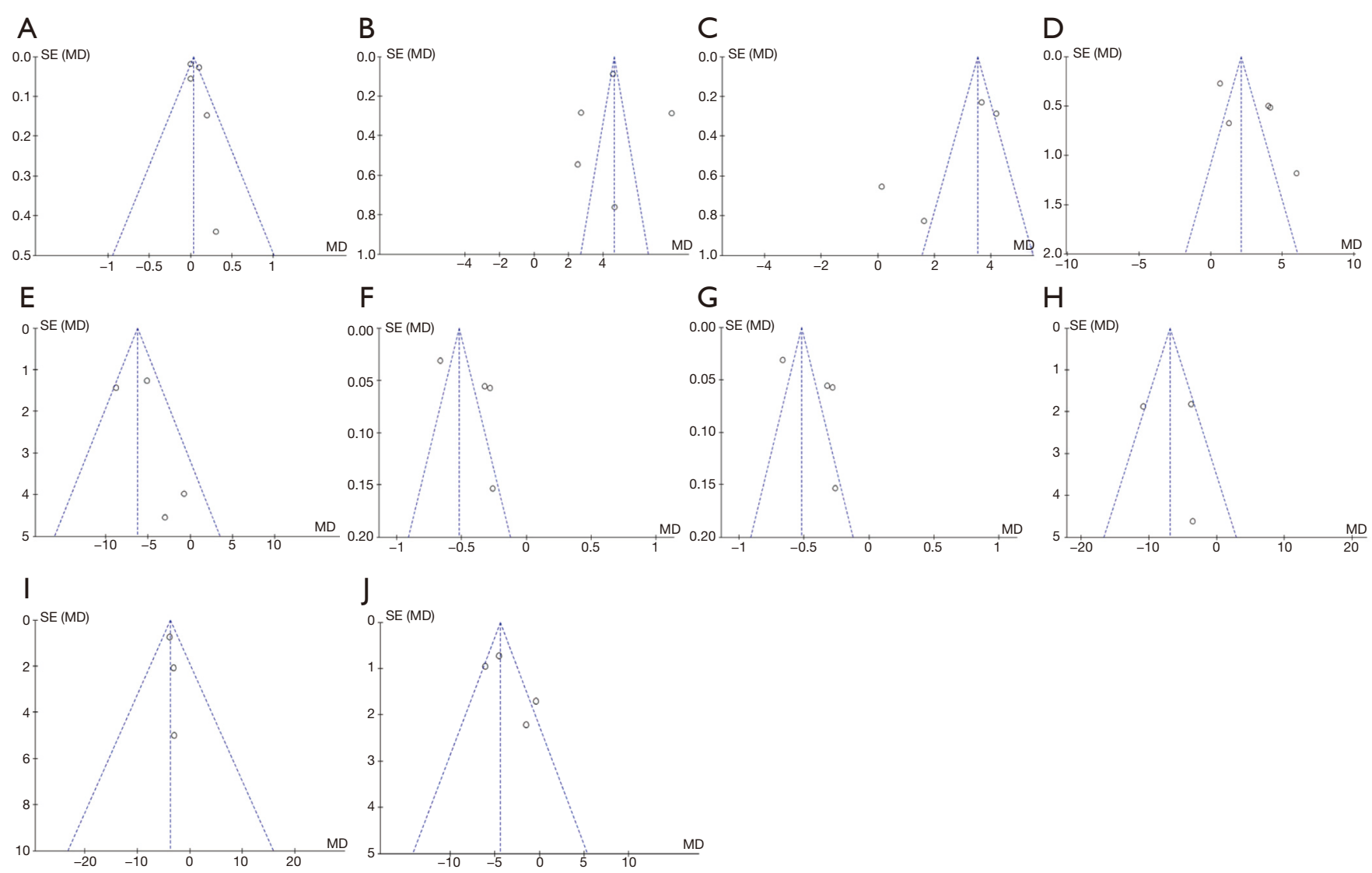

Figure 14 Funnel plots of various evaluation indicators. (A) totCa/ionCa; (B) prothrombin time; (C) activated partial thromboplastin time; (D) thrombin time; (E) platelet count; (F) cystatin; (G) alanine aminotransferase; (H) aspartate aminotransferase; (I) creatinine; (J) total bilirubin. totCa/ionCa, total calcium/ionized calcium; SE, standard error; $\mathrm{MD}$, mean difference.

liver and kidney function (27). Therefore, there is a clinical need to find effective anticoagulant treatment methods that can be applied to the CRRT process to ensure the treatment effect of patients (28). Correct selection of anticoagulants and close clinical monitoring are very important to prevent complications.

At present, heparin, a commonly used clinical anticoagulant, is combined with antithrombin-III to inhibit filter coagulation, prolong the use time of the filter, and achieve ideal anticoagulation effects (29). However, the incidence of adverse reactions is high, and it can often lead to thrombocytopenia, which increases the risk of bleeding in patients (30). Citric acid is a tricarboxylic acid organic compound, and its anticoagulant effect is related to calcium ions. Studies have shown that citrate can be injected into the arterial end of the cardiopulmonary bypass which binds calcium ions in the plasma to achieve anticoagulation. Therefore, when RCA is used, calcium chloride needs to be injected into the vein to replace the calcium ions lost in the blood (31). Citrate has little effect on the coagulation system, can improve the biocompatibility of the dialysis circuit, and reduce the patient's inflammatory response. Ordinary heparin-free dialysis can't effectively avoid the appearance of coagulation in extracorporeal circulation pipeline. RCA can be applied to patients with highrisk bleeding tendency and effectively prevent bleeding and other complications because it has no influence on the coagulation function in patients. Local in vitro anticoagulation of heparin, citrate, and low molecular weight heparin can be effectively applied in the treatment of CRRT in critically ill patients, and enhanced monitoring can reduce the occurrence of complications. To further study the clinical efficacy and coagulation function of RCA in the CRRT treatment of critically ill patients, the clinical RCTs on RCA for CRRT in critically ill patients were screened from the literature. In this study, ten randomized 
controlled clinical trials of CRRT critically ill patients with RCA were screened for meta-analysis. The clinical efficacy of anticoagulant citrate in CRRT in critically ill patients was quantitatively and systematically analyzed. The coagulation indicators of the patients in the experimental group were improved after treatment. The anticoagulant effect of citrate was similar to that of low molecular weight heparin, but compared with low molecular weight heparin, the anticoagulant effect of citrate was lower in bleeding risk, had less effect on coagulation function, and extended the service life of the filter. Relevant studies have revealed that citric acid used as an anticoagulant drug for hemodialysis can effectively reduce the risk of bleeding in patients during treatment and can increase the length of time the filter is used, which was similar to the results of this study.

\section{Conclusions}

There were 10 articles included in this meta-analysis of the clinical efficacy and coagulation function of RCA in the CRRT treatment of severely ill patients, involving 1,411 severely ill CRRT patients. The meta-analysis showed that RCA can more greatly improve the clinical efficacy and coagulation indicators of patients relative to heparin. However, this study still has certain limitations, including the large publication bias. In addition, due to different research directions, the sample size was small, the analytical indicators included were not comprehensive, and the results of meta-analysis weren't very accurate. Therefore, it is still necessary to select large sample size and high-quality RCA clinical effects for verification in future work.

\section{Acknowledgments}

Funding: None.

\section{Footnote}

Reporting Checklist: The authors have completed the PRISMA reporting checklist. Available at https://dx.doi. org/10.21037/apm-21-1693

Conflicts of Interest: All authors have completed the ICMJE uniform disclosure form (available at https://dx.doi. org/10.21037/apm-21-1693). The authors have no conflicts of interest to declare.

Ethical Statement: The authors are accountable for all aspects of the work in ensuring that questions related to the accuracy or integrity of any part of the work are appropriately investigated and resolved.

Open Access Statement: This is an Open Access article distributed in accordance with the Creative Commons Attribution-NonCommercial-NoDerivs 4.0 International License (CC BY-NC-ND 4.0), which permits the noncommercial replication and distribution of the article with the strict proviso that no changes or edits are made and the original work is properly cited (including links to both the formal publication through the relevant DOI and the license). See: https://creativecommons.org/licenses/by-nc-nd/4.0/.

\section{References}

1. Hoff BM, Maker JH, Dager WE, et al. Antibiotic dosing for critically ill adult patients receiving intermittent hemodialysis, prolonged intermittent renal replacement therapy, and continuous renal replacement therapy: an update. Ann Pharmacother 2020;54:43-55.

2. Trotman RL, Williamson JC, Shoemaker DM, et al. Antibiotic dosing in critically ill adult patients receiving continuous renal replacement therapy. Clin Infect Dis 2005;41:1159-66.

3. Rewa OG, Villeneuve PM, Lachance P, et al. Quality indicators of continuous renal replacement therapy (CRRT) care in critically ill patients: a systematic review. Intensive Care Med 2017;43:750-63.

4. Lewis SJ, Mueller BA. Antibiotic dosing in critically ill patients receiving CRRT: underdosing is overprevalent. Semin Dial 2014;27:441-5.

5. Ulldemolins $M$, Vaquer S, Llauradó-Serra M, et al. Betalactam dosing in critically ill patients with septic shock and continuous renal replacement therapy. Crit Care 2014;18:227.

6. Shotwell MS, Nesbitt R, Madonia PN, et al. Pharmacokinetics and pharmacodynamics of extended infusion versus short infusion piperacillin-tazobactam in critically ill patients undergoing CRRT. Clin J Am Soc Nephrol 2016;11:1377-83.

7. Pistolesi V, Morabito S, Di Mario F, et al. A guide to understanding antimicrobial drug dosing in critically ill patients on renal replacement therapy. Antimicrob Agents Chemother 2019;63:e0583-19.

8. Chen H, Yu RG, Yin NN, et al. Combination of extracorporeal membrane oxygenation and continuous renal replacement therapy in critically ill patients: a 
systematic review. Crit Care 2014;18:675.

9. Schultheiß C, Saugel B, Phillip V, et al. Continuous venovenous hemodialysis with regional citrate anticoagulation in patients with liver failure: a prospective observational study. Crit Care 2012;16:R162.

10. Lahmer T, Messer M, Rasch S, et al. Sustained lowefficiency dialysis with regional citrate anticoagulation in medical intensive care unit patients with liver failure: A prospective study. J Crit Care 2015;30:1096-100.

11. Slowinski T, Morgera S, Joannidis M, et al. Safety and efficacy of regional citrate anticoagulation in continuous venovenous hemodialysis in the presence of liver failure: the Liver Citrate Anticoagulation Threshold (L-CAT) observational study. Crit Care 2015;19:349.

12. Sponholz C, Settmacher U, Bauer M, et al. Regional citrate anticoagulation for continuous renal replacement therapy in the perioperative care of liver transplant recipients: a single center experience. Ther Apher Dial 2015;19:8-15.

13. Durão MS, Monte JC, Batista MC, et al. The use of regional citrate anticoagulation for continuous venovenous hemodiafiltration in acute kidney injury. Crit Care Med 2008;36:3024-9.

14. De Vico P, Messino V, Tartaglione A, et al. Safety and efficacy of citrate anti-coagulation continuous renal replacement therapies in post-cardiac surgery patients with liver dysfunction. Ther Apher Dial 2015;19:272-8.

15. Saner FH, Treckmann JW, Geis A, et al. Efficacy and safety of regional citrate anticoagulation in liver transplant patients requiring post-operative renal replacement therapy. Nephrol Dial Transplant 2012;27:1651-7.

16. Balogun RA, Turgut F, Caldwell S, et al. Regional citrate anticoagulation in critically ill patients with liver and kidney failure. J Nephrol 2012;25:113-9.

17. Klingele M, Stadler T, Fliser D, et al. Long-term continuous renal replacement therapy and anticoagulation with citrate in critically ill patients with severe liver dysfunction. Crit Care 2017;21:294.

18. Yu Y, Peng S, Cen Z, et al. Applying regional citrate anticoagulation in continuous renal replacement therapy for acute kidney injury patients with acute liver dysfunction: a retrospective observational study. Kidney Blood Press Res 2018;43:1065-74.

19. Broeker A, Wicha SG, Dorn C, et al. Tigecycline in critically ill patients on continuous renal replacement therapy: a population pharmacokinetic study. Crit Care 2018;22:341.

20. Chen G, Zhou Y, Ma J, et al. Is there a role for blood purification therapies targeting cytokine storm syndrome in critically severe COVID-19 patients? Ren Fail 2020;42:483-8.

21. Al-Hwiesh A, Abdul-Rahman I, Finkelstein F, et al. Acute kidney injury in critically ill patients: a prospective randomized study of tidal peritoneal dialysis versus continuous renal replacement therapy. Ther Apher Dial 2018;22:371-9.

22. Griffin BR, Thomson A, Yoder M, et al. Continuous renal replacement therapy dosing in critically ill patients: a quality improvement initiative. Am J Kidney Dis 2019;74:727-35.

23. Muilwijk EW, de Lange DW, Schouten JA, et al. Suboptimal dosing of fluconazole in critically ill patients: time to rethink dosing. Antimicrob Agents Chemother 2020;64:e00984-20.

24. Heintz BH, Matzke GR, Dager WE. Antimicrobial dosing concepts and recommendations for critically ill adult patients receiving continuous renal replacement therapy or intermittent hemodialysis. Pharmacotherapy 2009;29:562-77.

25. Premuzic V, Basic-Jukic N, Jelakovic B, et al. Differences in CVVH vs. CVVHDF in the management of sepsisinduced acute kidney injury in critically ill patients. J Artif Organs 2017;20:326-34.

26. Jonckheer J, Vergaelen K, Spapen H, et al. Modification of nutrition therapy during continuous renal replacement therapy in critically ill pediatric patients: a narrative review and recommendations. Nutr Clin Pract 2019;34:37-47.

27. Villa G, Neri M, Bellomo R, et al. Nomenclature for renal replacement therapy and blood purification techniques in critically ill patients: practical applications. Crit Care 2016;20:283.

28. Philpott CD, Droege CA, Droege ME, et al. Pharmacokinetics and pharmacodynamics of extendedinfusion cefepime in critically ill patients receiving continuous renal replacement therapy: a prospective, open-label study. Pharmacotherapy 2019;39:1066-76.

29. Raza A, Estepa A, Chan V, et al. Acute renal failure in critically ill COVID-19 patients with a focus on the role of renal replacement therapy: a review of what we know so far. Cureus 2020;12:e8429.

30. Kiser TH, Fish DN, Aquilante CL, et al. Evaluation of sulfobutylether- $\beta$-cyclodextrin (SBECD) accumulation and voriconazole pharmacokinetics in critically ill patients undergoing continuous renal replacement therapy. Crit Care 2015;19:32.

31. Meersch M, Küllmar M, Wempe C, et al. Regional citrate versus systemic heparin anticoagulation for continuous 
renal replacement therapy in critically ill patients with acute kidney injury (RICH) trial: study protocol for a multicentre, randomised controlled trial. BMJ Open 2019;9:e024411.

(English Language Editor: C. Betlazar-Maseh)

Cite this article as: Chang $\mathrm{H}$, Gong Y, Li C, Ma Z. Clinical efficacy of regional citrate anticoagulation in continuous renal replacement therapy: systematic review and meta-analysis. Ann Palliat Med 2021;10(8):8939-8951. doi: 10.21037/apm-21-1693 